\title{
Closed Facebook groups and COVID-19: an evaluation of utilization prior to and during the pandemic
}

\author{
Salvatore Docimo Jr. ${ }^{1}$ (1) $\cdot$ Brian Jacob $^{2} \cdot$ Kevin Seras $^{3} \cdot$ Omar Ghanem $^{4}$
}

Received: 6 June 2020 / Accepted: 27 August 2020 / Published online: 14 September 2020

(c) Springer Science+Business Media, LLC, part of Springer Nature 2020

\begin{abstract}
Background Surgical education was limited during the COVID-19 pandemic due to redeployment, limited clinical activity, and cancelation of elective procedures and educational conferences. Closed Facebook groups became a tool for surgical education while upholding social distancing guidelines. We aim to evaluate the use of Online Society of American Gastrointestinal and Endoscopic Surgeons (SAGES) closed Facebook groups, during and prior to the COVID-19 pandemic.

Methods Institutional Review Board evaluation and written consent was not indicated as the data does not pertain to any human subjects. Data files pertaining to new membership activity, posts, comments and reactions of eight closed Facebook groups. The pandemic group was defined as March 19th to April 30, 2020. The pre-pandemic group was defined as February 6th, to March 18th, 2020. The percentage increase of new memberships, posts, comments and reactions were calculated for each period. A two-tailed $t$-test, using a significance level of 0.05 was used to evaluate significance.

Results A statistically significant increase in membership during the pandemic period was noted for each group. In regards to posts, the Flex Endo, Acute Care, Colorectal, Foregut, and Bariatric groups were noted to have a statistically significant increase in the pandemic period. Colorectal and Bariatric groups were the only two groups that were noted to have a significant increase in comments in the pandemic period. For reactions, Flex Endo, Colorectal, Foregut, and Bariatric groups were noted to have experienced a significant increase during the pandemic.

Conclusions The COVID-19 pandemic halted surgical education at all levels. The membership and utilization of closed Facebook groups increased significantly in many instances, demonstrating the importance of internet-based surgical education now and into the future. Further development of internet-based curriculums is warranted.
\end{abstract}

Keywords Surgical education · COVID-19 and surgical education · Internet-based surgical education · Coronavirus and surgical education $\cdot$ Pandemic and surgical education

The field of surgery requires a life-long commitment to continued education and evolution in both knowledge and surgical technique. The process of surgical education in the modern era has been perpetually evolving from the traditional adage of "See one, do one, teach one" described by Sir William

Salvatore Docimo Jr.

Salvatore.docimo@stonybrookmedicine.edu

1 Division of Bariatric, Foregut, and Advanced Gastrointestinal Surgery, Renaissance School of Medicine at Stony Brook University, Stony Brook, NY, USA

2 Icahn School of Medicine at Mount Sinai, New York, NY, USA

3 Henry Ford Health Systems, Detroit, MI, USA

4 Minimally Invasive and Bariatric Surgery, Mosaic Life Care, University of Missouri, St Joseph, MO, USA
Halsted [1]. One sign of this evolution includes the multimedia approach to surgical education which involves a combination of text, graphics, audio, animation, and video. Proven benefits of this platform include improved surgical performance and the conversion of cognitive input into long-term memory [2, 3].

The use of online video training for surgical resident training has seen an increase in recent years [4]. The use of video and multimedia surgical education would be expected to increase during times of a pandemic. Surgical education has been limited in recent months due to COVID-19 with alterations such as redeployment, limited clinical activity, and cancelation of elective procedures and educational conferences. The American Board of Surgery has recognized this as they have modified training requirements for the graduating chief residents including a $10 \%$ reduction in total required cases [5]. 
Although this response is warranted in times of national crisis, it is not a substitute for the lost experience at all levels of surgical education. Many residencies across the USA have attempted to continue surgical education using non-traditional means, such as video lectures, live virtual didactics and independent learning [6]. Practicing surgeons also seek the opportunity to advance their surgical knowledge and techniques as well as a platform to discuss complex cases. Facebook groups, in which membership is restricted to practicing surgeons, residents, fellows, medical students, and certain members of the medical device industry [3], are one source of surgical education through social media. We aim to evaluate the use of Online Society of American Gastrointestinal and Endoscopic Surgeons (SAGES) closed Facebook groups, during and prior to the COVID-19 pandemic.

\section{Methods}

The data files regarding new membership activity, posts, comments, and reactions of eight closed Facebook groups were evaluated. The closed Facebook groups enrolled for evaluation were: Flex Endo, Acute Care Surgery, HPB Surgery, Robotic Surgery, Hernia Surgery, Colorectal Surgery, Foregut Surgery, and Bariatric Surgery.

The data were collected for the months of February 2020 through April 2020 using built in Facebook parameters that track and collect user data. The eight closed Facebook groups were then divided into two time periods: the pandemic group and the pre-pandemic group. The pandemic group was defined as the timeframe of March 19, 2020 to April 30, 2020. March 19th was chosen as the beginning of the pandemic phase as it marks the first date a state, California, enacted a stay-at-home order due to the COVID-19 pandemic1. The pre-pandemic group was defined as February 6, 2020 to March 18, 2020 as this marks the same amount of days as the pandemic group prior to the first stay-at-home order of March 19, 2020.

\section{Statistical analysis}

The percentage increase of new memberships, posts, comments and reactions were calculated for the pre-pandemic and pandemic groups for the each Facebook group. A two-tailed $t$-test was used to evaluate significance between the pre-pandemic and pandemic group, using a significance level of 0.05 .

Table 2 Comments and reactions for each Facebook group in the pre- and post-pandemic period

\begin{tabular}{|c|c|c|c|c|c|c|c|c|}
\hline & \multicolumn{4}{|l|}{ Comments } & \multicolumn{4}{|l|}{ Reactions } \\
\hline & Pre-pandemic & Pandemic & $\%$ Change & $p$-value & Pre-pandemic & Pandemic & $\%$ Change & $p$-value \\
\hline Flex Endo & $2.55 \pm 6.62$ & $4.28 \pm 14.40$ & 40.42 & 0.480 & $3.26 \pm 3.92$ & $7.16 \pm 13.03$ & 54.47 & 0.01 \\
\hline Acute Care & $5.04 \pm 8.68$ & $4.10 \pm 6.89$ & -22.93 & 0.61 & $7.41 \pm 12.66$ & $9.80 \pm 11.31$ & 24.39 & 0.407 \\
\hline HPB & $7.83 \pm 10.20$ & $11.28 \pm 25.24$ & 30.59 & 0.414 & $14.14 \pm 15.10$ & $27.46 \pm 49.55$ & 48.51 & 0.099 \\
\hline Robotic & $3.11 \pm 5.66$ & $1.81 \pm 4.42$ & -71.82 & 0.239 & $6.35 \pm 10.88$ & $4.27 \pm 8.03$ & -48.71 & 0.318 \\
\hline Hernia & $1.85 \pm 7.76$ & $0.28 \pm 0.77$ & -560.71 & 0.18830 & $2.74 \pm 10.88$ & $1.05 \pm 1.56$ & -160.95 & 0.316 \\
\hline Colorectal & $26.55 \pm 18.78$ & $53.60 \pm 62.56$ & 50.47 & 0.009 & $50.26 \pm 40.10$ & $99.81 \pm 89.28$ & 49.64 & 0.001 \\
\hline Foregut & $35.02 \pm 29.89$ & $40.74 \pm 47.16$ & 14.04 & 0.507 & $47.33 \pm 38.43$ & $71.89 \pm 54.17$ & 34.16 & 0.018 \\
\hline Bariatric & $13.24 \pm 14.83$ & $38.28 \pm 63.61$ & 65.41 & 0.015 & $16.07 \pm 17.19$ & $79.05 \pm 116.70$ & 79.67 & 0.0009 \\
\hline
\end{tabular}

Bold values are statistically significant for $p$-value

Table 1 Average membership and posts for each Facebook group in the pre- and post-pandemic period

\begin{tabular}{|c|c|c|c|c|c|c|c|c|}
\hline & \multicolumn{4}{|c|}{ Average membership } & \multicolumn{4}{|l|}{ Posts } \\
\hline & Pre-pandemic & Pandemic & $\%$ Change & $p$-value & Pre-pandemic & Pandemic & $\%$ Change & $p$-value \\
\hline Flex Endo & $647.98 \pm 11.40$ & $745.42 \pm 83.14$ & 13.07 & 0.00001 & $0.26 \pm 0.54$ & $0.86 \pm 1.22$ & 69.77 & 0.005 \\
\hline Acute Care & $1198.52 \pm 23.75$ & $1340.10 \pm 93.32$ & 10.56 & 0.00001 & $0.69 \pm 0.93$ & $1.37 \pm 1.31$ & 49.64 & 0.018 \\
\hline HPB & $1403.00 \pm 8.64$ & $1535.72 \pm 131.32$ & 8.64 & 0.00001 & $1.12 \pm 1.34$ & $1.62 \pm 1.86$ & 30.86 & 0.195 \\
\hline Robotic & $1574.40 \pm 44.94$ & $1736.30 \pm 107.00$ & 9.32 & 0.00001 & $0.76 \pm 0.91$ & $0.62 \pm 0.90$ & -22.58 & 0.495 \\
\hline Hernia & $1209.54 \pm 222.25$ & $1385 \pm 86.85$ & 12.67 & 0.00001 & $0.76 \pm 1.00$ & $0.53 \pm 0.71$ & -43.40 & 0.231 \\
\hline Colorectal & $2603.17 \pm 7.41$ & $2801.98 \pm 175.92$ & 7.10 & 0.00001 & $2.37 \pm 1.62$ & $4.26 \pm 2.42$ & 44.37 & 0.00006 \\
\hline Foregut & $2555.98 \pm 7.85$ & $2693 \pm 116.81$ & 5.09 & 0.00001 & $2.71 \pm 2.00$ & $4.23 \pm 3.68$ & 35.93 & 0.019 \\
\hline Bariatric & $1457.38 \pm 7.08$ & $1566.81 \pm 101.50$ & 6.98 & 0.00001 & $1.21 \pm 1.44$ & $3.37 \pm 3.37$ & 64.09 & 0.0003 \\
\hline
\end{tabular}

Bold values are statistically significant for $p$-value 


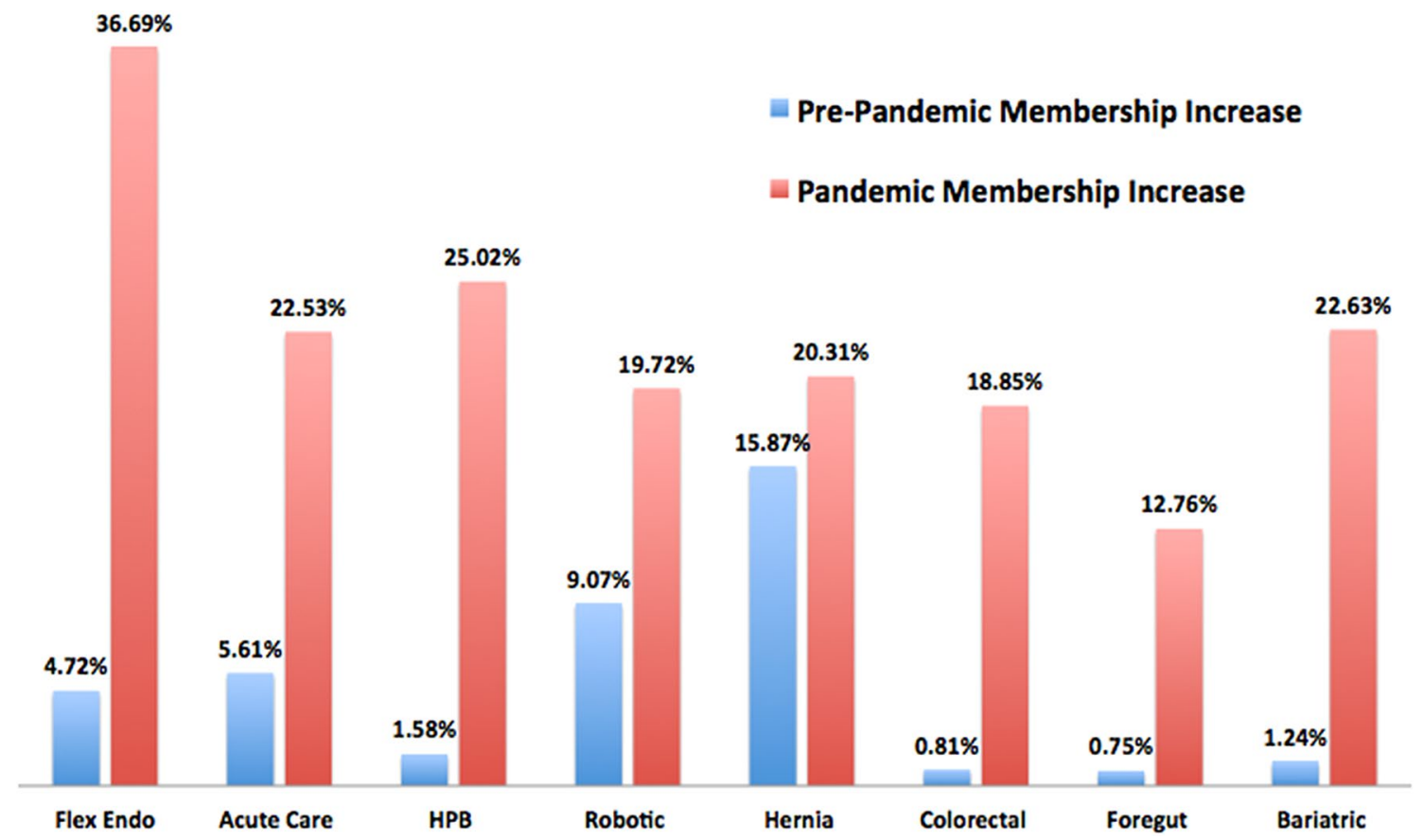

Fig. 1 Membership increase for each Facebook group for the pre- and post-pandemic period

\section{Results}

Eight SAGES closed Facebook groups were evaluated. Each group was noted to have a statistically significant increase in membership during the pandemic period. Tables 1 and 2 demonstrate the mean membership, posts, comments, and reactions for each Facebook group broken down by pre-pandemic and pandemic periods. Figure 1 demonstrates the percentage increase of membership for the pre-pandemic and pandemic groups. Each Facebook group, except for the robotic and hernia group, was noted to have a percentage increase in posts, comments, and reactions during the pandemic period compared to the pre-pandemic period as noted in Fig. 2.

In regards to posts, the Flex Endo, Acute Care, Colorectal, Foregut, and Bariatric groups were noted to have a statistically significant increase in the pandemic period. Colorectal and Bariatric groups were the only two groups that were noted to have a significant increase in comments in the pandemic period compared to the pre-pandemic group. For reactions, Flex Endo, Colorectal, Foregut, and Bariatric groups were noted to have experienced a significant increase during the pandemic.

\section{Discussion}

The present study demonstrates the significant use of online teaching modalities during the COVID-19 pandemic. In many instances, the standard interactions of live lectures and intra-operative experiences came to a halt during the pandemic. During the period of March 19, 2020 to April 30,2020 , the number of members joining the closed Facebook groups increased significantly and the use of the closed Facebook groups, such as posts, comments and reactions also increased significantly in some cases. Our findings demonstrate a significant demand and need for surgical education during the quarantine, more importantly; it also emphasized the increased use of online teaching modalities in a time of need.

Internet-based surgical curriculums have been successfully implemented previously in the undergraduate surgical education setting. A survey of 227 medical students $(78 \%$ response rate) demonstrated that $89 \%$ (mean rate of 4.5 out of 5.0) of medical students believe internet tools could be effectively applied in surgical education, specifically with a focus on lectures, assignments, real-time procedural demonstrations, case discussion, and interactions with surgical experts, with the benefits being a regular self-assessment (mean rating $=4.6$ ) and a more flexible learning schedule (mean rating $=4.0)$ [7].

Lead by Brian Jacob and SAGES leadership, the closed Facebook groups initiative, based on the SAGES Masters Program, was launched in March of 2017 [2]. The closed Facebook groups offer surgical trainees and practicing surgeons access to live-lectures and also case discussions which provide free, archivable, real-time opinions from experts throughout the world. The online lectures certainly can be further organized into a formal curriculum for trainees and 


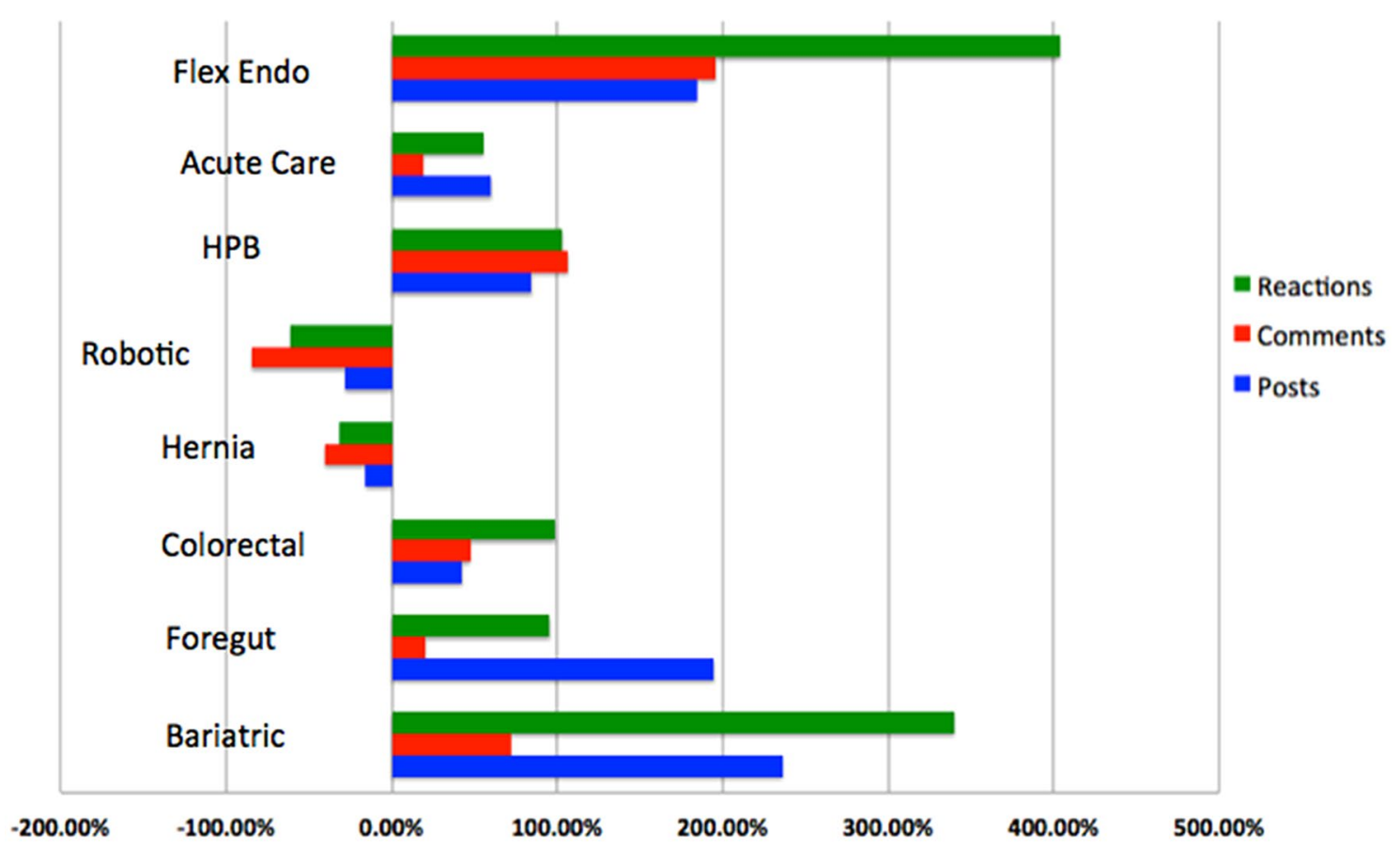

Fig. 2 Percentage changes for reactions, comments, and posts for each Facebook group for the pre- and post-pandemic period

also surgeons in practice. Once surgeons enter practice, they can experience a performance plateau and require continuing medical education (CME) to ensure active learning annually [2]. Such internet-based curriculums can act as sources for continued education and CME credit.

Between May 2017 and January 2018, the International Hernia Collaborative, a closed Facebook group that allows international surgeons to post clinical questions and exchange feedback, piloted a series of expert lectures followed by an interactive Facebook Live session, along with a pathway to earn CME credit. Ghanem et al. [8] noted an average of $1116(20.4 \pm 4.0 \%)$ viewed each live session event following each lecture $(n=9$ events). The average Facebook engagement score (defined as [(the number of comments $\times 2)+($ the number of reactions) $)$, was $259 \pm 75$, a significant difference with the average Facebook engagement score on the IHC (40.8) over the same time period $(p<0.001)$. On average, 16 users [range 8-35, $(n=7$ events $)]$ claimed CME credit for each educational series.

The positive findings of such pilot programs are instrumental in building the foundation for future social mediabased CME programs. Such internet-based curriculums will morph into a resource for surgeons to obtain CME credit. As Ghanem et al. [8] noted, the American Medical Association (AMA) modified their requirements for accredited CME providers to certify activities for AMA PRA Category 1 Credit. One such modification is the addition of an "other activity" format that does not fit within one of the previously existing CME formations [9]. It is likely a social media curriculum and online case discussion could fall within this "other activity" category.

Certainly the COVID-19 pandemic has created a unique environment and need for online medical education. At the time of this writing, six national meetings have been postponed (including the Society of American Gastrointestinal and Endoscopic Surgeons Annual Meeting), 26 have been switched to a virtual meeting, and 14 have been canceled. Considering a significant portion of these meetings have been moved to a virtual setting, it is not inconceivable for closed Facebook groups to become the foundation upon which future virtual meetings are based upon.

Panahi et al. [10] did identify six challenges facing physician adoption of social media: maintaining confidentiality, lack of active participation, finding time, lack of trust, workplace acceptance, and support and information anarchy. All Facebook groups stress the importance of posting no identifiable information, with posts being observed by forum moderators and administrators for any infractions, as private Facebook posts have been noted to be discoverable [11]. As noted by our data, a decrease in live patient and educational interactions due to the pandemic translated into an increase in active participation. As Fig. 2 demonstrates, the percentage increase for posts, comments, and reactions were increased in all Facebook groups except the robotic and hernia groups. The existence of two well-established closed Facebook groups, the International Hernia Collaborative (9600 members) and the Robotic Surgery Collaboration (11,000 members) 
may explain the decrease in activity during the COVID-19 pandemic period for their SAGES Facebook counterparts. The remaining findings can be attributed to both a need for surgical education and an increase in "free-time" due to pandemic lockdowns. Most importantly, the Facebook format allows members to access the closed Facebook groups $24 \mathrm{~h}$ a day, 7 days a week,

Limitations of this study do exist. Though we did demonstrate an increased usage of the Facebook platform, we did not evaluate the change in content, if any, during the COVID-19 pandemic. Furthermore, we did not evaluate if the increased usage of the Facebook platform created meaningful content retention (Fig. 2).

\section{Conclusion}

The COVID-19 pandemic created a unique circumstance by which surgical education at all levels ceased. The membership and utilization of closed Facebook groups increased significantly in many instances, demonstrating the importance of internet-based surgical education now and into the future. Further development of internet-based curriculums is warranted.

Acknowledgements We would like to thank Jason Levine, webmaster of the SAGES Facebook groups for his assistance during the data collection process.

\section{Compliance with ethical standards}

Disclosures Dr. Jacob reports Grants from Medtronic, Personal Fees from Medtronic, other from International Hernia Collaborative, outside the submitted work. Dr. Docimo reports Consultant Fees from Boston Scientific. Drs. Ghanem and Seeras have no conflicts of interest or financial ties to disclose.

\section{References}

1. Debes AJ, Aggarwal R, Balasundaram I, Jacobsen MB (2010) A tale of two trainers: virtual reality versus a video trainer for acquisition of basic laparoscopic skills. Am J Surg 199(6):840-845

2. Jones D, Stefanidis D, Korndorffer JR, Dimick JB, Jacob BP, Schultz L, Scott DJ (2017) SAGES University MASTERS Program: a structured curriculum for deliberate, lifelong learning. Surg Endosc 31(11):4863

3. Jackson HT, Young MT, Rodriguez AJ, Wright AS (2018) SAGES Foregut Surgery Masters Program: a surgeon's social media resource for collaboration, education, and professional development. Surg Endosc 32:2800-2807

4. Mendez A, Seikaly H, Ansari K et al (2014) High definition video teaching module for learning neck dissection. J Otolaryngol Head Neck Surg 43:7

5. https://www.absurgery.org/default.jsp?news_covid19_trainingre q. Accessed 5 June 2020

6. Coe TM, Jogerst KM, Sell NM et al (2020) Practical techniques to adapt surgical resident education to the COVID-19 era. Ann Surg. https://doi.org/10.1097/SLA.0000000000003993 (published online ahead of print, 29 April 2020)

7. Ekenze SO, Okafor CI, Ekenze OS, Nwosu JN, Ezepue UF (2017) The value of internet tools in undergraduate surgical education: perspective of medical students in a developing country. World $\mathbf{J}$ Surg 41(3):672-680

8. Ghanem O, Logghe HJ, Tran BV, Huyn D, Jacob B (2019) Closed Facebook $^{\mathrm{TM}}$ groups and CME credit: a new format for continuing medical education. Surg Endosc 33:587-591

9. American Medical Association (2017) The AMA's physician's recognition award and credit system. https://www.ama-assn.org/ sites/default/files/media-browser/public/cme/pra-booklet_0pd

10. Panahi S, Watson J, Partridge H (2016) Social media and physicians: exploring the benefits and challenges. Health Inform J 22(2):99-112

11. Fogelson NS, Rubin ZA, Ault KA (2013) Beyond likes and tweets: an in-depth look at the physician social media landscape. Clin Obstet Gynecol 56(3):495-508

Publisher's Note Springer Nature remains neutral with regard to jurisdictional claims in published maps and institutional affiliations. 